\title{
ON CANONICITY AND COMPLETIONS OF WEAKLY REPRESENTABLE RELATION ALGEBRAS
}

\author{
IAN HODKINSON AND SZABOLCS MIKULÁS
}

\author{
Abstract. We show that the variety of weakly representable relation algebras is neither canonical nor \\ closed under Monk completions.
}

$\S 1$. Introduction. The class wRRA of weakly representable relation algebras was introduced by Jónsson in [19]. It is obtained by dropping the requirement on classical representations of relation algebras that,+- are respected. It forms a 'half-way house' between the class RA of relation algebras and the class RRA of representable relation algebras, and is part of the currently rather active field of reducts of relation algebras. Although its notion of representation is weaker, it appears that in various ways, wRRA is at least as complex as RRA, but it has been less studied. More work is needed to elucidate its properties, and the current paper is a contribution to this.

Our aim here is to show that wRRA is not closed under taking canonical extensions or Monk completions. We believe that the arguments and constructions used to establish these results are at least as interesting as the results themselves, and that they may add to the currently rather limited stock of useful techniques for dealing with wRRA.

First we will recall the basic definitions and summarize some of the known results about wRRA. We refer the reader to [15] for notations of operations and classes of algebras. We will often identify (notationally) a structure, algebra, or graph with its domain. In Section 2, we briefly outline some aspects of canonical extensions and completions pertaining to wRRA. In Sections 3 and 4 we prove our main results that wRRA is neither a canonical class nor closed under completions. In Section 5 we state some open problems.

1.1. The class wRRA. It will be convenient to define weak representations by a first-order theory. Our definition is equivalent to the standard ones.

\section{Received August 31, 2010}

The first author offers his grateful thanks to Rob Goldblatt and Ed Mares in Victoria University of Wellington, New Zealand, for their generous hospitality during his visit in January 2010 when the research on this paper was begun. The authors also thank Rob Goldblatt for his questions and for valuable comments on the work, and the referees for very helpful remarks. This research was partially supported by grant VUW0503 from the Marsden Fund of the Royal Society of New Zealand. 
Definition 1.1. A weak representation of a relation algebra $\mathscr{A}=(A,+,-, \cdot$, $0,1,1,,, ;)$ is a structure $M$ whose signature consists of the elements of $A$, each a binary relation symbol, and satisfying, for each $r, s \in A$ :

1. $\forall x y(r \cdot s(x, y) \leftrightarrow r(x, y) \wedge s(x, y))$,

2. $\forall x y\left(1^{\prime}(x, y) \leftrightarrow x=y\right)$,

3. $\forall x y(\breve{r}(x, y) \leftrightarrow r(y, x))$,

4. $\forall x y(r ; s(x, y) \leftrightarrow \exists z(r(x, z) \wedge s(z, y)))$,

5. $\exists x y r(x, y)$ if $r \neq 0$,

6. $\forall x y \neg 0(x, y)$.

A relation algebra is said to be weakly representable if it has a weak representation. The class of weakly representable relation algebras is denoted by wRRA.

It is plain that RRA $\subseteq$ wRRA $\subseteq$ RA. There are no conditions in Definition 1.1 concerning + or - , and these operations are not required to be respected in a weak representation, though they are operations on the algebra and must conform to the relation algebra axioms (as given in, e.g., [15, Definition 3.8] and [22, §6]).

1.2. Existing results. There is not a great deal of literature about weakly representable relation algebras. We summarise some of it now, in order to place the main topic of our paper in some context.

1. In his original paper [19], Jónsson gave an infinite quasi-equational theory $\Gamma$ that defines wRRA in RA. He also showed that not every relation algebra is weakly representable, by constructing an (infinite) atomic $\mathscr{A} \in \mathrm{RA} \backslash$ wRRA.

2. In [1], Andréka constructed algebras in wRRA $\backslash R R A$, an ultraproduct of which is in RRA. Hence, RRA is not finitely axiomatisable over wRRA.

3. [18] used a so-called 'rainbow construction' to show that wRRA is not finitely axiomatisable.

4. [15, Theorem 18.23] used another rainbow construction to show that, restricted to isomorphism types of finite algebras, RRA and (the complement of) wRRA are recursively inseparable. It follows [15, Corollary 18.25] that $\mathrm{RA}_{n} \nsubseteq$ wRRA for each finite $n \geq 3$. ( $\mathrm{RA}_{n}$ is the class of " $n$-dimensional relation algebras': see [21] and [22, $\$ 6.24]$. The $\mathrm{RA}_{n}$ are 'approximations' to RRA in that $\mathrm{RA}=\mathrm{RA}_{4} \supset \mathrm{RA}_{5} \supset \cdots$ and $\bigcap_{n>4} \mathrm{RA}_{n}=\mathrm{RRA}$.)

5. Recently, Pécsi [25] showed that wRRA is closed under homomorphic images and is consequently a variety, answering a 50-year-old question of [19].

6. [16] constructs a finite weakly representable relation algebra that is not in $\mathrm{RA}_{n}$ for any finite $n \geq 5$. (The construction is also rainbow-style and is the basis of the one in the current paper.) So wRRA $\not \mathrm{RA}_{n}$, and the converse inclusion also fails (as mentioned above). For $n=5$, this answered a question of Maddux.

There is another class of algebras related to wRRA in [19], namely the class $\mathrm{R}\left(\cdot, 1^{\prime},,, ;\right)$ of subreducts of RRA. That is, we take subalgebras of the $\left\{\cdot, 1^{\prime},{ }^{\prime}, ;\right\}-$ reducts of representable relation algebras. The behaviour of $R(\cdot, 1,,, ;)$ is slightly different from that of wRRA. For instance, [2] shows that $R(\cdot, 1,,, ;)$ is only a quasi-variety, since it is not closed under homomorphic images. Furthermore, the equational theory of $\mathrm{R}(\cdot, 1,,, ;)$ is decidable [2] in contrast to the undecidability of the equational theory of wRRA, while neither $\mathrm{R}(\cdot, 1,,$, ;) nor the variety generated by it is finitely axiomatisable [12, 13, 18]. 
§2. Canonical extensions and completions. Canonical extensions of boolean algebras with additional operators (BAOs) were introduced by Jónsson and Tarski in $[20, \S 2]$. We assume some familiarity with them, but we will quickly reprise some salient points and establish some notation. For a BAO $\mathscr{A}$, let $\mathscr{A}_{+}$denote its dual space. This is the structure whose domain is the set of all ultrafilters of $\mathscr{A}$, and endowed with relations derived from the operations on $\mathscr{A}$. See [4, Definition 5.40] or [15, Definition 2.68] for details. With some abuse of terminology, a structure in this relational signature will be called an atom structure. For an atom structure $\mathcal{S}$, we let $\mathcal{S}^{+}$denote the full complex algebra over $\mathcal{S}$ : see [4, Definition 5.21] or [15, Definition 2.65] for details. The canonical extension $\mathscr{A}^{\sigma}$ of $\mathscr{A}$ can then be taken as the full complex algebra over $\mathscr{A}_{+}$: that is, $\mathscr{A}^{\sigma}=\left(\mathscr{A}_{+}\right)^{+}$. Up to isomorphism, $\mathscr{A}$ is a subalgebra of $\mathscr{A}^{\sigma}$ [20]. A class of BAOs is said to be canonical if it is closed under taking canonical extensions.

Canonical extensions are analogous to the 'canonical models' used in modal logic to prove completeness theorems (see, e.g., [4, 11]). However, canonicity frequently does not easily yield completeness theorems for algebras of relations, because the canonical extension of an algebra is typically not an algebra of relations of the desired kind. Nonetheless, it can still be helpful in a number of ways to know that the canonical extension of a representable algebra is also representable. ${ }^{1}$ Several kinds of representable algebra do indeed have this property. For example, Monk proved that the class RRA of representable relation algebras is canonical (this is reported in [23] and the first published proof is in [21]), and canonicity of the variety RCA $_{n}$ of representable $n$-dimensional cylindric algebras $(n<\omega)$ is proved in [14, 3.1.108].

For a variety $\mathrm{V}$ of BAOs, a stronger condition than canonicity is that $\mathrm{V}$ is elementarily generated: that is, generated as a variety by the full complex algebras of the atom structures in some elementary class. Goldblatt proved in [8, Theorem 3.6.7] that if $\mathrm{V}$ is generated by the full complex algebras of the atom structures in a class that is closed under ultraproducts, then $V$ is canonical. Hence, every elementarily generated variety must be canonical. It turns out that many varieties of representable algebras of relations are elementarily generated. They include RRA and $\mathrm{RCA}_{n}$ for $n<\omega$; for this and more examples, see [10, 3].

Goldblatt recently asked whether wRRA is elementarily generated. Since a necessary condition for this to be true is that wRRA is canonical, we can resolve it negatively with Theorem 3.5 in this paper: wRRA is not canonical. This marks a notable difference between wRRA and many other classes of algebras of relations studied so far.

Somewhat related to canonical extensions are completions. The completion of an arbitrary BAO $\mathscr{A}$ is a complete BAO $\mathscr{B} \supseteq \mathscr{A}$ in which $\mathscr{A}$ is dense. Monk showed in [24] that any completely additive BAO $\mathscr{A}$ has a completion $\mathscr{A}$ that is unique up to isomorphism. If $\mathscr{A}$ is atomic, $\mathscr{A}$ is isomorphic to the full complex algebra over the atom structure of $\mathscr{A}$ (see, e.g., [15, Definition 2.62] for atom structures of atomic BAOs). It was proved in [17] that RRA and $\mathrm{RCA}_{n}$ for finite $n \geq 3$ are not closed under completions. Here, we will prove the same for wRRA, in Theorem 3.6 - in fact it follows from [6, Theorem 3.6] and the non-canonicity of wRRA. In this respect, wRRA is more in line with other classes of algebras of relations.

${ }^{1}[20$, p.892] makes similar points. 
$\S 3$. Non-canonicity of wRRA. We will show that the weakly representable relation algebras do not form a canonical class, by constructing a relation algebra $\mathscr{A} \in$ wRRA whose canonical extension $\mathscr{A}^{\sigma}$ is not in wRRA. That wRRA is not closed under Monk completions follows from its non-canonicity, but we will also show it directly using an algebra similar to $\mathscr{A}$.

$\mathscr{A}$ is a variant of the relation algebra constructed in [16]. To control whether it is weakly representable or not, we will construct it from a graph. (For other relation algebras based on graphs, see, e.g., [15, chapter 14].) In the next Section (3.1) we recall some basic definitions related to graphs $\Gamma$, in Section 3.2 we describe how to construct relation algebras $\mathscr{A}(\Gamma)$, and in Section 3.3 we establish some relationships between $\Gamma$ and $\mathscr{A}(\Gamma)$. Then in Section 3.4 we will be ready to state the main results. The most technical elements of the proof are presented in Section 4.

3.1. Graphs. Graphs here are undirected and loop-free, so formally a graph is a structure $\Gamma=(G, E)$, where $G$ is a non-empty set of nodes and $E$ is an irreflexive and symmetric binary relation on $G$. The edges of $\Gamma$ are the pairs $x y$ of nodes such that $(x, y) \in E$. We identify (notationally) a graph $\Gamma$ with its set of nodes.

For a graph $\Gamma$, a node $x \in \Gamma$, and a subset $X \subseteq \Gamma$, we write $E(x)$ for the set $\{y \in \Gamma: x y$ is an edge of $\Gamma\}$ of 'neighbours' of $x$, and $E(X)=\bigcup_{x \in X} E(x)$. (There will be no need to write $E_{\Gamma}(x)$, etc., as the ambient graph will always be clear from the context.) The set $X$ is independent if for each $x, y \in X, x y$ is not an edge. Let $\chi(\Gamma)$ denote the chromatic number of $\Gamma$, i.e., the smallest natural number $n$ such that $\Gamma$ is the union of $n$ independent sets. If no such $n$ exists, we let $\chi(\Gamma)=\infty$.

For nodes $x, y \in \Gamma$, a path from $x$ to $y$ is a sequence $\left(x_{1}, x_{2}, \ldots, x_{n}\right)$ of nodes such that $x_{1}=x, x_{n}=y$, and $x_{1} x_{2}, \ldots, x_{n-1} x_{n}$ are edges. $\Gamma$ is connected if there is a path between any two distinct nodes.

For finite $n \geq 3$, a cycle of length $n$ in $\Gamma$ is here taken to be a path $\left(x_{1}, x_{2}, \ldots\right.$, $\left.x_{n}, x_{1}\right)$ such that $x_{1}, \ldots, x_{n} \in \Gamma$ are pairwise distinct. It is well known (see, e.g., $[5$, Proposition 1.6.1]) that

$$
\chi(\Gamma) \leq 2 \text { iff } \Gamma \text { has no cycles of odd length. }
$$

Given graphs $\Gamma, \Delta$, a map $v: \Gamma \rightarrow \Delta$ is said to be a bounded morphism if for each $x \in \Gamma, v$ maps the set of neighbours of $x$ in $\Gamma$ surjectively onto the set of neighbours of $v(x)$ in $\Delta$.

3.2. The rainbow algebra $\mathscr{A}(\Gamma)$. Given any graph $\Gamma$, the 'rainbow' algebra $\mathscr{A}(\Gamma)$ is the full complex algebra $\alpha(\Gamma)^{+}$over the following atom structure $\alpha(\Gamma)$, which is a variant of one in [16]. The atoms are:

- $1^{\prime}, \mathrm{g}_{i}, \mathrm{w}_{i}, \breve{\mathrm{w}}_{i}(i \in\{0,1,2,3\}), \mathrm{v}, \mathrm{y}_{x}, \mathrm{r}_{x}(x \in \Gamma)$.

All atoms shown here are distinct. We regard the $w_{i}$ as white, the $g_{i}$ as green, the $\mathrm{y}_{x}$ as yellow, and the $\mathrm{r}_{x}$ as red. (1', v, and the $\breve{\mathrm{w}}_{i}$ have no special colour.) $1^{\prime}$ is the sole identity atom. The converse of $\mathrm{w}_{i}$ is $\breve{\mathrm{w}}_{i}$, and vice versa (each $i<4$ ), and all other atoms are self-converse. Composition can be specified by listing the forbidden cycles $(a, b, c)$ of atoms - those such that $\breve{c} \not \leq a ; b$ in $\mathscr{A}(\Gamma)$ - and here they are:

F1. (1', $a, b)$ whenever $a \neq \breve{b}$,

F2. $\left(\mathrm{g}_{i}, \mathrm{~g}_{j}, \mathrm{~g}_{k}\right)$ for each pairwise distinct $i, j, k<4$,

F3. $\left(\mathrm{y}_{x}, \mathrm{y}_{y}, \mathrm{r}_{z}\right)$ for each pairwise distinct $x, y, z \in \Gamma$, 


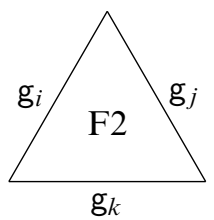

$i, j, k<4$ distinct.

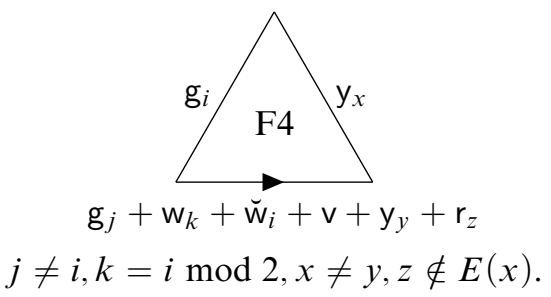

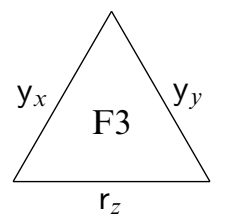

$x, y, z \in \Gamma$ distinct.

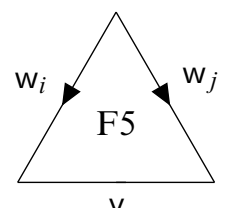

$i, j<4$ distinct.

FiguRE 1. The main forbidden cycles of $\mathscr{A}(\Gamma)$.

F4. $\left(\mathrm{y}_{x}, \mathrm{~g}_{i}, b\right)$ for each $x \in \Gamma, i<4$, and $b \in B_{x, i}$, where

$$
\begin{aligned}
B_{x, i}= & \left\{\mathrm{g}_{j}: j<4, j \neq i\right\} \cup\left\{\mathrm{w}_{k}: k<4, k=i \bmod 2\right\} \\
& \cup\left\{\breve{\mathrm{w}}_{i}, \mathrm{v}\right\} \cup\left\{\mathrm{y}_{y}: y \in \Gamma \backslash\{x\}\right\} \cup\left\{\mathrm{r}_{z}: z \in \Gamma \backslash E(x)\right\},
\end{aligned}
$$

F5. $\left(\check{\mathrm{w}}_{i}, \mathrm{w}_{j}, \mathrm{v}\right)$ for each distinct $i, j<4$.

We will refer to F1-F5 as rules. We stipulate that all Peircean transforms of cycles forbidden by a rule are also forbidden by the same rule: i.e., if $(a, b, c)$ is forbidden by a rule then so are $(b, c, a)$ and $(\breve{c}, \breve{b}, \breve{a})$. Figure 1 illustrates the rules other than F1. The extra-boolean operators and ; are now determined for all elements of $\mathscr{A}(\Gamma)$ using additivity.

It is plain that the algebras $\mathscr{A}(\Gamma)$ are BAOs. In Section 4 we will show that they are always relation algebras (Lemma 4.7), and give more information about them. In particular, we show that there is a strong connection between the chromatic number of $\Gamma$ and the weak representability of $\mathscr{A}(\Gamma)$ : roughly, that

$$
\chi(\Gamma)>2 \text { if and only if } \mathscr{A}(\Gamma) \in \text { wRRA. }
$$

See Propositions 4.8 and 4.9 for full details.

3.3. Duality. We now turn to the connection between maps on graphs, atom structures, and algebras. The following specialises [4, Definition 3.13] to atom structures $\alpha(\Gamma)$.

Definition 3.1. Let $\Gamma, \Delta$ be graphs. A map $f: \alpha(\Delta) \rightarrow \alpha(\Gamma)$ is a bounded morphism if

1. $f\left(1^{\prime}\right)=1^{\prime}$,

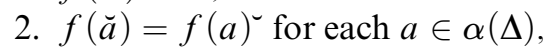

3. if the cycle $(a, b, c)$ is not forbidden in $\alpha(\Delta)$ then the cycle $(f(a), f(b), f(c))$ is not forbidden in $\alpha(\Gamma)$, 
4. if $a \in \alpha(\Delta), b^{\prime}, c^{\prime} \in \alpha(\Gamma)$, and the cycle $\left(f(a), b^{\prime}, c^{\prime}\right)$ is not forbidden in $\alpha(\Gamma)$, then there are $b, c \in \alpha(\Delta)$ such that $f(b)=b^{\prime}, f(c)=c^{\prime}$, and the cycle $(a, b, c)$ is not forbidden in $\alpha(\Delta)$.

Lemma 3.2. Any surjective bounded morphism $v: \Delta \rightarrow \Gamma$ of graphs induces a surjective bounded morphism $\alpha(v): \alpha(\Delta) \rightarrow \alpha(\Gamma)$ and an algebra embedding $\alpha(v)^{+}: \mathscr{A}(\Gamma) \rightarrow$ $\mathscr{A}(\Delta)$. The functionals $\alpha(-), \alpha(-)^{+}$preserve the identity and respect composition of maps.

Proof. Given a surjective bounded morphism $v: \Delta \rightarrow \Gamma$ of graphs, we may define $\alpha(v): \alpha(\Delta) \rightarrow \alpha(\Gamma)$ by $(\alpha(v))\left(\mathrm{y}_{x}\right)=\mathrm{y}_{v(x)},(\alpha(v))\left(\mathrm{r}_{x}\right)=\mathrm{r}_{v(x)}$, for each $x \in \Delta$, and $(\alpha(v))(a)=a$ for each $a \in \alpha(\Delta)$ that is neither yellow nor red. It is easy to verify that $\alpha(v)$ is a surjective bounded morphism in the sense of Definition 3.1. By general duality (see, e.g., [4, Proposition 5.51]), the map $\alpha(v)^{+}: \mathscr{A}(\Gamma) \rightarrow \mathscr{A}(\Delta)$ given by $\alpha(v)^{+}(S)=\{a \in \alpha(\Delta):(\alpha(v))(a) \in S\}$, for $S \subseteq \alpha(\Gamma)$, is an algebra embedding. The last part of the lemma is easy to verify.

We assume basic familiarity with direct and inverse systems and their limits. To fix notation, a direct (respectively inverse) system (over $(\omega, \leq)$ ) will be taken to be a family of the form $\left(D_{n}, \theta_{n}^{m}: n \leq m<\omega\right)$ (respectively, $\left(\bar{I}_{n}, \phi_{n}^{m}: n \leq m<\omega\right)$ ), where $D_{n}, I_{n}$ are structures of some kind, and for each $n \leq m \leq k<\omega, \theta_{n}^{m}: D_{n} \rightarrow$ $D_{m}$ and $\phi_{n}^{m}: I_{m} \rightarrow I_{n}$ are maps, $\theta_{n}^{n}$ is the identity map on $D_{n}, \phi_{n}^{n}$ is the identity map on $I_{n}, \theta_{n}^{k}=\theta_{m}^{k} \circ \theta_{n}^{m}$, and $\phi_{n}^{k}=\phi_{n}^{m} \circ \phi_{m}^{k}$.

Let $\mathscr{D}=\left(\mathscr{A}_{n}, \theta_{n}^{m}: n \leq m<\omega\right)$ be a direct system of BAOs and embeddings. Then by standard duality (see, e.g., [4, Theorem 5.47(iii)]),

$$
\mathscr{D}_{+}=\left(\left(\mathscr{A}_{n}\right)_{+},\left(\theta_{n}^{m}\right)_{+}: n \leq m<\omega\right)
$$

is an inverse system of atom structures and surjective bounded morphisms, where the maps $\left(\theta_{n}^{m}\right)_{+}$are defined in the obvious way by $\left(\theta_{n}^{m}\right)_{+}(\mu)=\left\{a \in \mathscr{A}_{n}: \theta_{n}^{m}(a) \in \mu\right\}$ for an ultrafilter $\mu \in\left(\mathscr{A}_{m}\right)_{+}$. Regarding atom structures as relational structures as usual, $\lim _{\leftarrow}\left(\mathscr{D}_{+}\right)$is a well defined atom structure. We now quote a consequence of results of Goldblatt [7, Theorems 10.7, 11.2, 11.6] :

FACT 3.3 (Goldblatt 1976). $\left(\lim _{\rightarrow} \mathscr{D}\right)_{+} \cong \underset{\leftarrow}{\lim }\left(\mathscr{D}_{+}\right)$.

We use this to derive the following proposition. The key part is part 4, which in our main argument (Theorem 3.5) will be applied to the inverse system $\mathscr{G}$ in (5) below.

Proposition 3.4. Let $\mathscr{G}=\left(\Gamma_{n}, v_{n}^{m}: n \leq m<\omega\right)$ be an inverse system of finite graphs and surjective bounded morphisms. In the notation of Lemma 3.2, define

$$
\begin{aligned}
\alpha(\mathscr{G}) & =\left(\alpha\left(\Gamma_{n}\right), \alpha\left(v_{n}^{m}\right): n \leq m<\omega\right), \\
\mathscr{A}(\mathscr{G}) & =\left(\mathscr{A}\left(\Gamma_{n}\right), \alpha\left(v_{n}^{m}\right)^{+}: n \leq m<\omega\right) .
\end{aligned}
$$

Then:

1. $\alpha(\mathscr{G})$ is an inverse system of atom structures and surjective bounded morphisms,

2. $\mathscr{A}(\mathscr{G})$ is a direct system of BAOs and embeddings,

3. $(\lim \mathscr{A}(\mathscr{G}))_{+} \cong \alpha(\lim \mathscr{G})$,

4. $\left(\lim _{\rightarrow} \mathscr{A}(\mathscr{G})\right)^{\sigma} \cong \mathscr{A}(\overleftarrow{\leftarrow} \underset{\leftarrow}{\leftarrow} \mathscr{G})$

\footnotetext{
${ }^{2}$ Goldblatt proved these results in the context of modal algebras. However, they generalise easily to BAOs.
} 
Proof. Parts 1 and 2 are immediate from Lemma 3.2. For part 3, first observe that $\alpha\left(\lim _{\leftarrow} \mathscr{G}\right) \cong \lim _{\leftarrow} \alpha(\mathscr{G})$ : this is easy to show. Now as each $\alpha\left(\Gamma_{n}\right)$ is finite, $\mathscr{A}\left(\Gamma_{n}\right)_{+} \cong \alpha\left(\Gamma_{n}\right)$, and this can be extended to show that $\alpha(\mathscr{G})$ is isomorphic to the inverse system $\mathscr{A}(\mathscr{G})_{+}$as defined in (3). By this and Fact $3.3, \lim _{\leftarrow} \alpha(\mathscr{G}) \cong$ $\lim _{\leftarrow}\left(\mathscr{A}(\mathscr{G})_{+}\right) \cong\left(\lim _{\rightarrow} \mathscr{A}(\mathscr{G})\right)_{+}$. Part 3 now follows, and part 4 is an immediate consequence.

\subsection{Main results.}

THEOREM 3.5. wRRA is not a canonical class.

Proof. In Section 3.2, we built a 'rainbow' algebra $\mathscr{A}(\Gamma)$ for each graph $\Gamma$. We will prove in Section 4 that

1. if $\Gamma$ is connected and $\chi(\Gamma)>2$, then $\mathscr{A}(\Gamma) \in$ wRRA: see Proposition 4.9,

2. if $|\Gamma| \geq 2$ and $\chi(\Gamma) \leq 2$ then $\mathscr{A}(\Gamma) \notin$ wRRA: see Proposition 4.8.

For each $0<n<\omega$, let $\Gamma_{n}$ be a graph consisting of a cycle of length $3^{n}$. Formally, we may let the nodes of $\Gamma_{n}$ be the integers $\bmod 3^{n}$, with edges relating elements differing by 1 :

$$
\Gamma_{n}=\left(\mathbb{Z} / 3^{n} \mathbb{Z},\left\{\left(3^{n} \mathbb{Z}+k, 3^{n} \mathbb{Z}+k+1\right),\left(3^{n} \mathbb{Z}+k, 3^{n} \mathbb{Z}+k-1\right): k \in \mathbb{Z}\right\}\right) .
$$

Each $\Gamma_{n}$ is connected and has chromatic number 3, so by the first point above,

$$
\mathscr{A}\left(\Gamma_{n}\right) \in \text { wRRA. }
$$

For $0<n \leq m<\omega$ we define a map $v_{n}^{m}: \Gamma_{m} \rightarrow \Gamma_{n}$ that 'wraps' $\Gamma_{m}$ onto $\Gamma_{n}$, by $v_{n}^{m}\left(3^{m} \mathbb{Z}+k\right)=3^{n} \mathbb{Z}+k(k \in \mathbb{Z})$. Then

$$
\mathscr{G}=\left(\Gamma_{n}, v_{n}^{m}: 0<n \leq m<\omega\right)
$$

is an inverse system of finite graphs and surjective bounded morphisms. By Proposition 3.4 and (4) above, $\mathscr{A}(\mathscr{G})$ is a direct system of weakly representable relation algebras and relation algebra embeddings. Let $\mathscr{A}=\lim _{\rightarrow} \mathscr{A}(\mathscr{G})$ be its direct limit. Then $\mathscr{A} \in$ wRRA, since wRRA is a variety [25] and so closed under direct limits.

Now by Proposition 3.4(4), $\mathscr{A}^{\sigma} \cong \mathscr{A}\left(\Gamma_{\omega}\right)$, where $\Gamma_{\omega}$ is the inverse limit of $\mathscr{G}$. But $\Gamma_{\omega}$ is the graph whose nodes are the 3-adic integers (see, e.g., [26] for information), with edges consisting of all pairs of 3 -adic integers that differ by 1 . Because the ring of 3-adic integers has characteristic zero, it is easy to see that $\Gamma_{\omega}$ has no cycles. Alternatively, assuming that $\left(x_{1}, \ldots, x_{k}, x_{1}\right)$ is a cycle in $\Gamma_{\omega}$, pick a finite $n \geq k$ such that the $x_{i}$ map to distinct elements under the natural projection from $\Gamma_{\omega}$ onto $\Gamma_{n}$. Then the image of $\left(x_{1}, \ldots, x_{k}, x_{1}\right)$ is a cycle of length $k$ in $\Gamma_{n}-$ impossible, since $\Gamma_{n}$ is a cycle of length $3^{n}$.

So by $(1), \chi\left(\Gamma_{\omega}\right) \leq 2$. Certainly, $\left|\Gamma_{\omega}\right| \geq 2$. By the second point above, $\mathscr{A}\left(\Gamma_{\omega}\right) \notin$ wRRA. We conclude that $\mathscr{A}^{\sigma} \notin$ wRRA while $\mathscr{A} \in$ wRRA, whence wRRA is not canonical.

We remark that $\mathscr{A} \notin \mathrm{RRA}$, because otherwise, by canonicity of RRA we would have $\mathscr{A}^{\sigma} \in \mathrm{RRA} \subseteq$ wRRA, contradicting the above. As RRA is a variety and so closed under direct limits, $\mathscr{A}\left(\Gamma_{n}\right) \notin$ RRA for all but at most finitely many $n<\omega$.

THEOREM 3.6. wRRA is not closed under Monk completions.

Proof. Given Theorem 3.5, this follows from [6, Theorem 3.6], where it is shown that any universal class of monotone lattice expansions that is closed under completions is canonical. 
We can also exhibit a weakly representable relation algebra whose completion is not weakly representable. Let $\Gamma_{n}(1 \leq n<\omega)$ be as in Theorem 3.5. We will see in Proposition 4.9 that $\mathscr{A}\left(\Gamma_{n}\right) \in$ wRRA for each $n$. Let $D$ be a non-principal ultrafilter over $\{1,2, \ldots\}$, and let $\mathscr{A}$ be the ultraproduct $\prod_{D} \mathscr{A}\left(\Gamma_{n}\right)$. As wRRA is an elementary class, $\mathscr{A} \in$ wRRA.

Let $\Gamma=\prod_{D} \Gamma_{n}$. It is easily seen that $\mathscr{A}$ is atomic with atom structure $\prod_{D} \alpha\left(\Gamma_{n}\right) \cong$ $\alpha(\Gamma)$. Hence the Monk completion $\overline{\mathscr{A}}$ of $\mathscr{A}[24]$ is isomorphic to $\mathscr{A}(\Gamma)$. Since the property of having a cycle of a given length $k$ is expressible by a first-order sentence, it follows by Łośs theorem that $\Gamma$ has no cycles, and so by $(1)$ we have $\chi(\Gamma) \leq 2$. By Proposition 4.8, $\mathscr{A}(\Gamma) \notin$ wRRA.

§4. Weak representability. In this, the most technical section, we establish the two results on weak representability, Propositions 4.8 and 4.9, referred to in the proofs of Theorems 3.5 and 3.6.

4.1. Networks and games. Let $\mathscr{A}$ be a relation algebra. We will give a sufficient condition for $\mathscr{A}$ to be weakly representable, involving a game played on networks.

Definition 4.1. A network (over $\mathscr{A}$ ) is a pair $N=\left(N_{1}, N_{2}\right)$, where $N_{1} \neq \emptyset$ is a set of 'nodes', and $N_{2}: N_{1} \times N_{1} \rightarrow \mathscr{A}$ is a 'labelling function' satisfying, for all $x, y, z \in N_{1}$,

1. $N_{2}(x, y) \leq 1$ ' iff $x=y$,

2. $N_{2}(x, y)=N_{2}(y, x)^{-}$,

3. $0<N_{2}(x, y) \leq N_{2}(x, z) ; N_{2}(z, y)$.

For networks $N=\left(N_{1}, N_{2}\right), N^{\prime}=\left(N_{1}^{\prime}, N_{2}^{\prime}\right)$, we write $N \subseteq N^{\prime}$ if $N_{1} \subseteq N_{1}^{\prime}$ and $N_{2}^{\prime} \uparrow\left(N_{1} \times N_{1}\right)=N_{2}$. For a network $N=\left(N_{1}, N_{2}\right)$, we will usually drop the suffixes and write $N$ for any of $N, N_{1}, N_{2}$, distinguishing the meaning by context.

Definition 4.2. Players $\forall, \exists$ play a game $G(\mathscr{A})$ of length $\omega$ as follows. In an initial un-numbered round, $\forall$ chooses a nonzero element $a \in \mathscr{A}$, and $\exists$ responds with a network $N_{0}$ containing nodes $x, y$ with $N_{0}(x, y) \leq a$. In round $l<\omega$, if the current network (at the start of the round) is $N_{l}$, then $\forall$ chooses nodes $x, y \in N_{l}$ and elements $a, b \in \mathscr{A}$ with $a ; b \geq N_{l}(x, y) . \exists$ must respond with a network $N_{l+1} \supseteq N_{l}$ containing a node $z$ with $N_{l+1}(x, z) \leq a$ and $N_{l+1}(z, y) \leq b . \exists$ wins the game if she always responds to $\forall$ with such a network.

The following lemma was proved for finite relation algebras in [16, Proposition 10], in which case the converse is also true. This would suffice for our purposes (recall that each $\mathscr{A}\left(\Gamma_{n}\right)$ from Section 3.4 is finite), but we prove it now for arbitrary relation algebras for completeness.

Lemma 4.3. Let $\mathscr{A}$ be a relation algebra. If $\exists$ has a winning strategy in $G(\mathscr{A})$ then $\mathscr{A}$ is weakly representable.

Proof. Suppose that $\exists$ has a winning strategy in $G(\mathscr{A})$. First consider the case where $\mathscr{A}$ is countable. For each nonzero $a \in \mathscr{A}$, consider a play $N_{0} \subseteq N_{1} \subseteq \cdots$ of the game in which $\forall$ plays $a$ initially and arranges to play subsequently every move that ever becomes possible during play (he can do this because $\mathscr{A}$ is countable), and in which $\exists$ uses her winning strategy. Let $N^{a}$ be the set of nodes occurring in networks in this play. For $x, y \in N^{a}$ and $b \in \mathscr{A}$, define $N^{a} \models b(x, y)$ iff there is 
$n<\omega$ with $x, y \in N_{n}$ and $N_{n}(x, y) \leq b$. Then the disjoint union of the $N^{a}$, taken over all nonzero elements $a \in \mathscr{A}$, is a weak representation of $\mathscr{A}$.

Now let $\mathscr{A}$ have arbitrary cardinality. We may fix a winning strategy $\sigma$ for $\exists$ in $G(\mathscr{A})$ that provides a unique finite network (i.e., with finitely many nodes) for her to play in any situation. We build a chain $\mathscr{A}_{0} \preceq \mathscr{A}_{1} \preceq \cdots \preceq \mathscr{A}$ of countable elementary subalgebras of $\mathscr{A}$. To begin, let $\mathscr{A}_{0}$ be an arbitrary countable elementary subalgebra of $\mathscr{A}$. Given $\mathscr{A}_{n}$, let $\mathscr{A}_{n+1}$ be any countable elementary subalgebra of $\mathscr{A}$ containing $\mathscr{A}_{n}$ and such that for any play of $G(\mathscr{A})$ in which $\forall$ only chooses elements of $\mathscr{A}_{n}$ and in which $\exists$ uses $\sigma$, all networks played are networks over $\mathscr{A}_{n+1}$. Let $\mathscr{A}_{\omega}=\bigcup_{n<\omega} \mathscr{A}_{n}$. Then $\mathscr{A}_{\omega} \preceq \mathscr{A}$, and $\exists$ has a winning strategy in $G\left(\mathscr{A}_{\omega}\right)$. As $\mathscr{A}_{\omega}$ is countable, $\mathscr{A}_{\omega} \in$ wRRA, and as wRRA is elementary we obtain $\mathscr{A} \in$ wRRA as required.

4.2. Basic properties of $\mathscr{A}(\Gamma)$. Next we state some properties of $\mathscr{A}(\Gamma)$ that will be useful later on. First we define the following elements of $\mathscr{A}(\Gamma)$ :

- $0^{\prime}=-1^{\prime}$ (as usual in relation algebras),

- $\mathrm{G}=\sum_{i<4} \mathrm{~g}_{i}$,

- $\mathrm{W}=\sum_{i<4} \mathrm{~W}_{i}$,

- $\mathrm{r}_{X}=\sum\left\{\mathrm{r}_{x}: x \in X\right\}$ and $\mathrm{y}_{X}=\sum\left\{\mathrm{y}_{x}: x \in X\right\}$, for any $X \subseteq \Gamma{ }^{3}$

Lemma 4.4. If $a, b \in \mathscr{A}(\Gamma)$ satisfy $0<a, b \leq 0^{\prime}$, then $(a ; b) \cdot \mathrm{W}>0$.

Proof. It is clear that none of F2-F5 alone can block all the white atoms. So $(a ; b) \cdot \mathrm{W}>0$ for any atoms $a, b \leq 0$. The result for arbitrary elements follows by additivity of composition.

Lemma 4.5. If $\Gamma$ is connected and has at least two nodes, and $a, b \in \mathscr{A}(\Gamma)$ satisfy $0<a, b \leq 0^{\prime}$, then $(a ; b) \cdot \mathrm{r}_{\Gamma}>0$.

Proof. This is because no rule other than F1 can bar all red atoms. We give a little more detail. By additivity of composition, we can assume that $a, b$ are atoms. Let $z \in \Gamma$. If $\mathrm{r}_{z} \leq a ; b$, we are done. If not, it is because of rule $\mathrm{F} 3$ or $\mathrm{F} 4$. If the former, we have $a=\mathrm{y}_{x}$ and $b=\mathrm{y}_{y}$ for some distinct $x, y \neq z$. But then $\mathrm{r}_{x} \leq a ; b$. If the latter, we have $a=\mathrm{g}_{i}$ and $b=\mathrm{y}_{x}$ (or vice versa) for some $i<4$ and $x \in \Gamma$ with $z \notin E(x)$. But $\Gamma$ is connected with at least two nodes, so $E(x) \neq \emptyset$. Then $\mathrm{r}_{y} \leq a ; b$ for any $y \in E(x)$.

Lemma 4.6. If $a, b \in \mathscr{A}(\Gamma)$ satisfy $0<a, b \leq 0$, then $a ; b \geq a+b$.

Proof. Again, we may assume that $a, b$ are atoms. Observe that no cycle forbidden by a rule other than F1 involves an atom and its converse. ${ }^{4}$ Consequently, the cycles $(a, b, \breve{a})$ and $(a, b, \breve{b})$ are not forbidden, and so $a ; b \geq a+b$ as required.

LEMma 4.7. $\mathscr{A}(\Gamma)$ is a relation algebra.

Proof. By (the proof of) [15, Lemma 3.24], it is enough to prove that the atom structure $\alpha(\Gamma)$ of $\mathscr{A}(\Gamma)$ has the following three properties:

1. For all $x, y \in \alpha(\Gamma)$ we have $x=y$ iff the cycle $\left(1^{\prime}, x, \breve{y}\right)$ is not forbidden,

2. all Peircean transforms of a forbidden cycle are forbidden,

\footnotetext{
${ }^{3}$ To ensure that this is well defined, we suppose $\Gamma \cap \wp(\Gamma)=\emptyset$.

${ }^{4} \mathrm{~F} 1$ does forbid some such cycles: e.g., $\left(1^{\prime}, \breve{1}, v\right)$.
} 


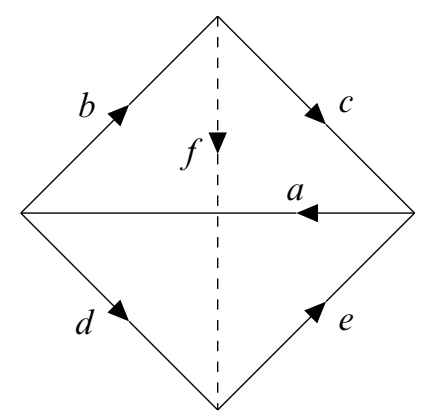

FIGURE 2. The atoms $a, b, c, d, e$.

3. for all $a, b, c, d, e \in \alpha(\Gamma)$, if $(b, c, a)$ and $(d, e, a)$ are not forbidden cycles, then there is some atom $f$ such that the cycles $(b, f, \breve{d})$ and $(f, e, \breve{c})$ are not forbidden either. See figure 2.

The first property follows from $\mathrm{F} 1$ and the second is immediate from the definition. The third property is easily proved if $1^{\prime} \in\{b, c, d, e\}$. Consider for example the case where $b=1$ '. Let $f=d$. Then obviously $(b, f, \breve{d})=\left(1^{\prime}, d, \breve{d}\right)$ is not forbidden. Moreover, as $(b, c, a)$ is not forbidden, by $\mathrm{F} 1$ we have $a=\breve{c}$, so $(f, e, \breve{c})=(d, e, a)$, which is assumed not to be forbidden. The other cases are proved similarly.

If $1^{\prime} \notin\{b, c, d, e\}$ then inspection of $\mathrm{F} 2-\mathrm{F} 5$ shows that there can be at most three atoms $x \leq \mathrm{W}+\breve{W}$ such that $(b, x, \breve{d})$ is forbidden, and at most three atoms $y \leq \mathrm{W}+\breve{\mathrm{W}}$ such that $(y, e, \breve{c})$ is forbidden. There are eight atoms $f \leq \mathrm{W}+\breve{\mathrm{W}}$, so for at least two of them, $(b, f, \breve{d})$ and $(f, e, \breve{c})$ are not forbidden.

4.3. Weak representability of $\mathscr{A}(\Gamma)$. We are ready to establish the main representability results about $\mathscr{A}(\Gamma)$.

Proposition 4.8. Suppose that $\Gamma$ is a graph with $\chi(\Gamma) \leq 2$ and $|\Gamma| \geq 2$. Then $\mathscr{A}(\Gamma) \notin$ wRRA.

Proof. Assume for contradiction that $M$ is a weak representation of $\mathscr{A}(\Gamma)$. Take $a, b \in M$ with $M \models \mathrm{v}(a, b)$. Since $\mathrm{v} \leq \mathrm{g}_{i} ; \mathrm{g}_{j}$ for all $i, j<4$, there exist $c, d \in M$ with $M \models \mathrm{g}_{0}(a, c) \wedge \mathrm{g}_{2}(c, b)$ and $M \models \mathrm{g}_{1}(a, d) \wedge \mathrm{g}_{3}(d, b)$. Then $M \models\left(\mathrm{g}_{0} ; \mathrm{g}_{1}\right) \cdot\left(\mathrm{g}_{2} ; \mathrm{g}_{3}\right)(c, d)$. Note that

$$
\left(\mathrm{g}_{0} ; \mathrm{g}_{1}\right) \cdot\left(\mathrm{g}_{2} ; \mathrm{g}_{3}\right)=0^{\prime}-\left(\mathrm{G}+\mathrm{y}_{\Gamma}\right) .
$$

Now take non-empty independent sets $P, Q \subseteq \Gamma$ with $P \cup Q=\Gamma$ and $P \cap Q=\emptyset$. These exist because $\chi(\Gamma) \leq 2$ and $|\Gamma| \geq 2$. We have

$$
\mathrm{y}_{P} ; \mathrm{y}_{Q}=0^{\prime}-\mathrm{G} \text {. }
$$

(E.g., if $x \in P$ and $y \in Q$ then $\mathrm{r}_{x}+\mathrm{r}_{y} \leq \mathrm{y}_{x} ; \mathrm{y}_{y}$, and it follows that $\mathrm{r}_{\Gamma} \leq \mathrm{y}_{P} ; \mathrm{y}_{Q}$.) Therefore, $\left(\mathrm{g}_{0} ; \mathrm{g}_{1}\right) \cdot\left(\mathrm{g}_{2} ; \mathrm{g}_{3}\right) \leq \mathrm{y}_{P} ; \mathrm{y}_{Q}$, so $M \models \mathrm{y}_{P} ; \mathrm{y}_{Q}(c, d)$ and there must be some $e \in M$ with $M \models \mathrm{y}_{P}(c, e) \wedge \mathrm{y}_{Q}(e, d)$. See figure 3 .

Clearly, $M \models\left(\mathrm{g}_{0} ; \mathrm{y}_{P}\right) \cdot\left(\mathrm{g}_{1} ; \mathrm{y}_{Q}\right)(a, e)$. What sum of atoms is $\left(\mathrm{g}_{0} ; \mathrm{y}_{P}\right) \cdot\left(\mathrm{g}_{1} ; \mathrm{y}_{Q}\right)$ ? Each atom in the sum lies beneath $\mathrm{g}_{0} ; \mathrm{y}_{P}$, so by F1, 1' is ruled out, and by F4, so are $\mathrm{g}_{1}, \mathrm{~g}_{2}, \mathrm{~g}_{3}, \mathrm{w}_{0}, \mathrm{w}_{2}, \breve{\mathrm{w}}_{0}, \mathrm{v}, \mathrm{y}_{y}$ for all $y \notin P$, and $\mathrm{r}_{z}$ for all $z \notin E(P)$. It also lies beneath $\mathrm{g}_{1} ; \mathrm{y}_{Q}$, and by $\mathrm{F} 4$ this additionally rules out $\mathrm{g}_{0}, \mathrm{w}_{1}, \mathrm{w}_{3}, \breve{\mathrm{w}}_{1}, \mathrm{y}_{y}$ for all $y \notin Q$, 


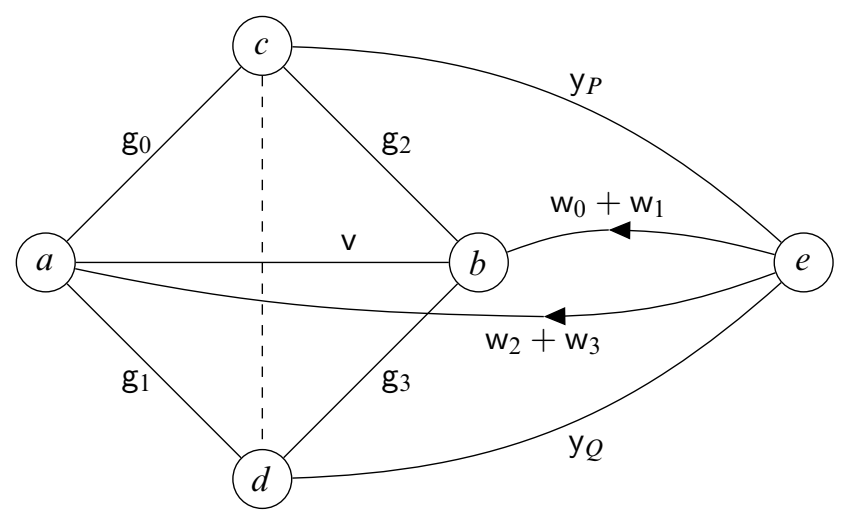

Figure 3. Points $a, b, c, d, e \in M$.

and $\mathrm{r}_{z}$ for all $z \notin E(Q)$. Since $P \cap Q=\emptyset$, all $\mathrm{y}_{y}$ are ruled out. Since $P, Q$ are independent and $P \cup Q=\Gamma$, we see that $E(P) \cap E(Q)=\emptyset$ and so all $\mathrm{r}_{z}$ are ruled out. All that remain are $\breve{\mathrm{w}}_{2}$ and $\breve{\mathrm{w}}_{3}$. So $\left(\mathrm{g}_{0} ; \mathrm{y}_{P}\right) \cdot\left(\mathrm{g}_{1} ; \mathrm{y}_{Q}\right)=\breve{\mathrm{w}}_{2}+\breve{\mathrm{w}}_{3}$ and we have $M \models\left(\breve{\mathrm{w}}_{2}+\breve{\mathrm{w}}_{3}\right)(a, e)$. Similarly, $M \models\left(\mathrm{w}_{0}+\mathrm{w}_{1}\right)(e, b)$. But now, by F5,

$$
\left(\left(\breve{\mathrm{w}}_{2}+\breve{\mathrm{w}}_{3}\right) ;\left(\mathrm{w}_{0}+\mathrm{w}_{1}\right)\right) \cdot \mathrm{v}=0,
$$

so $M \models 0(a, b)$, contradicting the last axiom defining weak representations.

Proposition 4.9. If $\Gamma$ is a connected graph with $\chi(\Gamma)>2$, then $\mathscr{A}(\Gamma) \in$ wRRA.

Proof. Write $\mathscr{A}$ for $\mathscr{A}(\Gamma)$ in the proof. By Proposition 4.7, $\mathscr{A}$ is a relation algebra. It remains to show that $\mathscr{A}$ is weakly representable, and for this, by Lemma 4.3 it suffices to show that $\exists$ has a winning strategy in $G(\mathscr{A})$.

$\exists$ will maintain two conditions during the game. To explain them, let $N$ be any network over $\mathscr{A}$. An edge of $N$ is a pair $(x, y)$ of nodes of $N$. Such an edge is said to be a critical edge of $N$ if $x \neq y$ and there are nodes $z, t \in N$, necessarily distinct from each other and from $x, y$, such that

- $N(z, t) \geq \mathrm{v}$,

- $N(x, z) \cdot \mathrm{G} \neq 0, N(x, t) \cdot \mathrm{G} \neq 0, N(y, z) \cdot \mathrm{G} \neq 0$, and $N(y, t) \cdot \mathrm{G} \neq 0$.

$\exists$ will ensure that each network played satisfies the following inductive conditions:

I1. $N(x, y) \leq 0$ ' for each distinct $x, y \in N$,

I2. $N(x, y) \geq \mathrm{r}_{\Gamma}$ for each critical edge $(x, y)$ of $N$.

In the initial round of $G(\mathscr{A}), \forall$ chooses a nonzero $\alpha \in \mathscr{A} . \exists$ chooses an atom $a \leq \alpha$, and responds with a network $N_{0}$ consisting of nodes $x, y$, say, where $x=y$ iff $a=1$ ', and with $N_{0}(x, y)=a$. This information is sufficient to determine $N_{0}$ completely, and it is clearly a network satisfying the inductive conditions I1 and I2.

Suppose that at the start of some later round of the game, the current network is $N$, and assume inductively that it satisfies I1 and I2. Let $\forall$ play the round by choosing nodes $m, n \in N$, say, and elements $\mu, v \in \mathscr{A}$ with $\mu ; v \geq N(m, n)$. Since $N$ is a network, $N(m, n)>0$, so $\mu, v>0$ as well. $\exists$ must come up with a network $N^{\prime} \supseteq N$ satisfying conditions I1 and I2 and containing a node $p$ with $N^{\prime}(m, p) \leq \mu$ and $N^{\prime}(p, n) \leq v$. So we are free to replace $\mu, v$ by smaller elements $\mu^{\prime} \leq \mu$ and 
$v^{\prime} \leq v$, so long as we keep the condition that $\mu^{\prime} ; v^{\prime} \geq N(m, n)$. We will do this in the claims below.

First, suppose that $m=n$ and $1^{\prime} \leq \mu \cdot v$. Then taking $p=m$, we have $N(m, p) \leq 1^{\prime} \leq \mu$ and $N(p, n) \leq 1^{\prime} \leq v . \exists$ may now simply respond with $N^{\prime}=N$, completing this round of the game. So from now on, we assume that this is not the case.

Clatm 1. We may assume that $\mu, v \leq 0$.

Proof of Claim. First, consider the case when $m=n$. As $N$ is a network and 1'

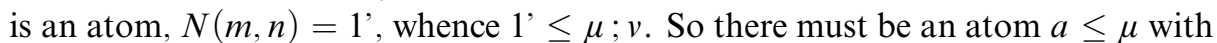
$\breve{a} \leq v$. By our recent assumption, $a \neq 1$ '. Since $N(m, n) \leq a$; $\breve{a}$, we may replace $\mu$ by $a$ and $v$ by $\breve{a}$. Then $\mu, v \leq 0$ as required. Hence in this case we can assume that $\mu, v$ are atoms and $v=\breve{\mu}$.

Now consider the case when $m \neq n$. Let $\mu=\mu_{0}+\mu_{1}$ where $\mu_{0} \leq 0^{\prime}$ and $\mu_{1} \leq 1$ '. Let $v=v_{0}+v_{1}$ similarly. Then

$$
\begin{array}{rlrl}
N(m, n) & \leq \mu ; v & \\
& =\left(\mu_{0}+\mu_{1}\right) ;\left(v_{0}+v_{1}\right) & \\
& =\mu_{0} ; v_{0}+\mu_{0} ; v_{1}+\mu_{1} ; v_{0}+\mu_{1} ; v_{1} & \\
& \leq \mu_{0} ; v_{0}+\mu_{0}+v_{0}+1, & & \text { as } \mu_{1}, v_{1} \leq 1 \\
& =\mu_{0} ; v_{0}+1, & & \text { by Lemma 4.6. }
\end{array}
$$

By inductive assumption I1, $N(m, n) \leq 0$, so in fact $N(m, n) \leq \mu_{0} ; v_{0}$. So we may replace $\mu$ by $\mu_{0}$ and $v$ by $v_{0}$. This proves the claim.

ClaIM 2. We may further assume that $\mu \leq N(m, n) ; \breve{v}$ and $v \leq \breve{\mu} ; N(m, n)$.

Proof of Claim. Let $C$ be the set of atoms $c$ of $\mathscr{A}(\Gamma)$ with $c \leq N(m, n)$. For each $c \in C$, select atoms $a_{c} \leq \mu$ and $b_{c} \leq v$ with $a_{c} ; b_{c} \geq c$. Let $\mu^{\prime}=\sum_{c \in C} a_{c}$ and $v^{\prime}=\sum_{c \in C} b_{c}$. Clearly, $\mu^{\prime} \leq \mu, v^{\prime} \leq v$, and $N(m, n)=\sum C \leq \sum_{c \in C} a_{c} ; b_{c} \leq$ $\mu^{\prime} ; v^{\prime}$. Also, for each $c \in C$ we have $a_{c} \leq c ; \breve{b}_{c} \leq N(m, n) ; \breve{v}^{\prime}$, so $\mu^{\prime} \leq N(m, n) ; \breve{v}^{\prime}$. Similarly, $v^{\prime} \leq \breve{\mu}^{\prime} ; N(m, n)$. So we may replace $\mu$ by $\mu^{\prime}$ and $v$ by $v^{\prime}$. This proves the claim. Note that if $m=n$ then $\mu, v$ are unchanged, as they are atoms.

$\exists$ now defines $N^{\prime}$ by adding a single new node to $N-$ say, $p$, with $p \notin N$. She defines the labelling of $N^{\prime}$ as follows. We must have $N^{\prime} \supseteq N$, so we only need specify labels $N^{\prime}(x, y)$ where $p \in\{x, y\}$. We also must have $N^{\prime}(y, x)=N^{\prime}(x, y)^{-}$, so we only need specify one of $N^{\prime}(x, y), N^{\prime}(y, x)$.

First, $\exists$ defines $N^{\prime}(m, p)=\mu, N^{\prime}(p, p)=1^{\prime}$, and $N^{\prime}(p, n)=v$. This is well defined, for if $m=n$ then $v=\breve{\mu}$ : see the proofs of the claims. Thus, our specifications of $N^{\prime}(p, n)=v$ and $N^{\prime}(p, n)=N^{\prime}(n, p)^{-}=N^{\prime}(m, p)^{-}=\breve{\mu}$ do not conflict.

Next, for each $q \in N \backslash\{m, n\}$, she defines $N^{\prime}(q, p)$ as follows, where we write $\alpha=N(q, m)$ and $\beta=N(q, n)$ :

$$
N^{\prime}(q, p)= \begin{cases}(\alpha ; \mu) \cdot(\beta ; \breve{v}) \cdot\left(\mathrm{r}_{\Gamma}+\mathrm{W}+\breve{\mathrm{W}}\right), & \text { if } \alpha \cdot \mathrm{G}>0 \text { and } \beta \cdot \mathrm{G}>0, \\ (\alpha ; \mu) \cdot(\beta ; \breve{v}) \cdot\left(\mathrm{r}_{\Gamma}+\mathrm{W}\right), & \text { otherwise. }\end{cases}
$$

Our job is to show that this defines a bona fide network satisfying the inductive conditions. It will occupy us for the rest of the section. 
By Claim 1 and the above Definition (6), inductive condition I1 clearly holds. We check that condition I 2 holds too. Let $(x, y)$ be a critical edge of $N^{\prime}$. So there are $z, t \in N^{\prime}$ with $N^{\prime}(z, t) \geq \mathrm{v}$ and such that $N^{\prime}(x, z), N^{\prime}(x, t), N^{\prime}(y, z)$, and $N^{\prime}(y, t)$ all contain green atoms. First suppose that $x, y \in N$. Then $z, t \in N$ as well because of the following. If, say, $z=p$, then there must be some $q \in\{x, y, t\} \cap(N \backslash\{m, n\})$, since $x, y, z, t$ are distinct. The label on $(q, z)=(q, p)$ is above either $v$ or a green atom. But $\exists$ 's strategy in (6) never defines such a label on edges of this form. The argument if $t=p$ is similar. Since $x, y, z, t \in N,(x, y)$ is a critical edge of $N$, and we conclude by inductive hypothesis I 2 that $N^{\prime}(x, y)=N(x, y) \geq \mathrm{r}_{\Gamma}$.

Now suppose that $(x, y)$ involves the new node $p$ : say without loss of generality that $x=p$. Again, as $N^{\prime}(p, z), N^{\prime}(p, t)$ are both above green atoms and $\exists$ 's strategy (6) only uses sums of atoms $\mathrm{r}_{x}, \mathrm{w}_{i}, \breve{\mathrm{w}}_{i}$ to label edges $(p, q)$ for $q \neq m, n$, we must have $\{z, t\}=\{m, n\}$. Hence, $y \in N \backslash\{m, n\}$. Inspection of (6) shows that $\exists$ would label $N^{\prime}(x, y)=N^{\prime}(y, x)^{-}$by a sum of atoms including all $\mathrm{r}_{w}$ such that $\mathbf{r}_{w} \leq(N(y, m) ; \mu) \cdot(N(y, n) ; \breve{v})$. Since $N(y, m), \mu, N(y, n), v$ all contain green atoms, and there are no forbidden cycles of the form $\left(\mathrm{g}_{i}, \mathrm{~g}_{j}, \mathrm{r}_{w}\right)$, we see that all $\mathrm{r}_{w}$ satisfy this condition. Hence $\mathrm{r}_{\Gamma} \leq N^{\prime}(x, y)$, which establishes inductive condition I2.

It remains to show that $N^{\prime}$ is a network. Conditions 1 and 2 of Definition 4.1 are easy to confirm, using the definitions and Claim 1 and remembering that $N \subseteq N^{\prime}$ and $N$ is a network. Next we check that all labels on edges of $N^{\prime}$ are nonzero. This is true for edges of $N$ since $N$ is a network. We have $N^{\prime}(m, p)=\mu$ and $N^{\prime}(p, n)=v$, and $\mu, v>0$ because $\mu ; v \geq N(m, n)>0$. Also, $N^{\prime}(p, p)=1^{\prime} \neq 0$. Let $q \in N \backslash\{m, n\}$. Denote $\alpha=N(q, m)$ and $\beta=N(q, n)$, and also write $\varepsilon$ for $N^{\prime}(q, p)$. See figure 4 .

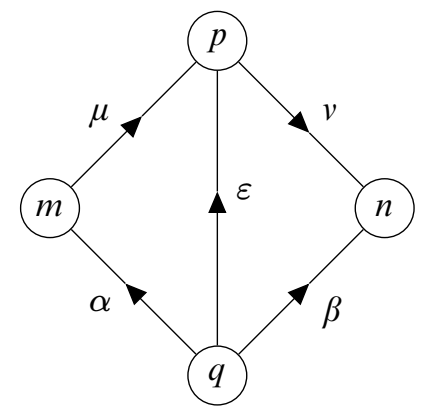

Figure 4. Points $m, n, p, q \in N^{\prime}$.

We show that $\varepsilon>0$. $\exists$ 's strategy in (6) always includes in $\varepsilon$ all atoms $\mathrm{r}_{x}, \mathrm{w}_{i}$ that can be consistently added. If $\varepsilon \cdot \mathrm{W}>0$, we are done. Suppose that $\varepsilon \cdot \mathrm{W}=0$. Only three rules can block white atoms, namely F1, F4, and F5. Rule F1 is not involved, since by I1 and Claim $1, \alpha, \beta, \mu, v \leq 0^{\prime}$. If $\mathrm{F} 5$ is involved, we must have $\mathrm{w}_{j} \leq \alpha$ for some $j$, and $\mathrm{v} \leq \mu-$ or alternatively, $\mathrm{w}_{j} \leq \beta$ for some $j$, and $\mathrm{v} \leq v$. Assume the latter; the former case is similar. But then $r_{\Gamma} \leq w_{j} ; v \leq \beta ; \breve{v}$. By Lemma 4.5, $\mathrm{r}_{\Gamma} \cdot(\alpha ; \mu)>0$, so by $(6), \varepsilon=N^{\prime}(q, p) \geq \mathrm{r}_{\Gamma} \cdot(\alpha ; \mu) \cdot(\beta ; \breve{v})>0$ as required. If instead only F4 is involved, in order to block all four $w_{i}$ we must certainly have 
$\mathrm{y}_{x} \leq \mu, \mathrm{y}_{y} \leq v, \mathrm{~g}_{i} \leq \alpha$, and $\mathrm{g}_{j} \leq \beta$, for some $x, y \in \Gamma$ and $i, j<4$. But then $\breve{\mathrm{w}}_{k} \leq\left(\mathrm{g}_{i} ; \mathrm{y}_{x}\right) \cdot\left(\mathrm{g}_{j} ; \mathrm{y}_{y}\right) \leq(\alpha ; \mu) \cdot(\beta ; \breve{v})$ for each $k<4$ with $k \neq i, j$, and $\exists$ 's strategy as defined in (6) would ensure that all such $\breve{w}_{k}$ are included in the label $N^{\prime}(q, p)$, which is consequently nonzero as required.

Labels on converse edges are the converses of the labels on the original edges, and so are also nonzero. This completes the check that all labels in $N^{\prime}$ are nonzero.

Our main work is to check that $N^{\prime}(x, y) \leq N^{\prime}(x, z) ; N^{\prime}(z, y)$ for every $x, y, z \in$ $N^{\prime}$. Since $N$ is a network, this is true if $x, y, z \in N$, so we may assume that $p \in\{x, y, z\}$. We also assume that $x, y, z$ are pairwise distinct, since otherwise, the statement is easy to prove using properties already established. We divide into cases according as $|\{x, y, z\} \cap\{m, n\}|=2,1$, or 0 .

First suppose that $\{x, y, z\}=\{m, n, p\}$. This case is an easy consequence of Claim 2 and the properties of converse.

Next, assume that $\{x, y, z\}=\{p, m, q\}$ for some $q \in N \backslash\{m, n\}$. Again let $\alpha=N(q, m), \beta=N(q, n)$, and $\varepsilon=N^{\prime}(q, p)$. It is clear by $\exists$ 's strategy that $\varepsilon \leq \alpha ; \mu$ — that is, $N^{\prime}(q, p) \leq N^{\prime}(q, m) ; N^{\prime}(m, p)$. That $N^{\prime}(p, q) \leq N^{\prime}(p, m) ; N^{\prime}(m, q)$ follows by taking converses. It remains to show that

$$
\alpha \leq \varepsilon ; \breve{\mu} \text { and } \mu \leq \breve{\alpha} ; \varepsilon \text {. }
$$

That is, $N^{\prime}(q, m) \leq N^{\prime}(q, p) ; N^{\prime}(p, m)$ and $N^{\prime}(m, p) \leq N^{\prime}(m, q) ; N^{\prime}(q, p)$. By taking converses we obtain $N^{\prime}(m, q) \leq N^{\prime}(m, p) ; N^{\prime}(p, q)$ and also $N^{\prime}(p, m) \leq$ $N^{\prime}(p, q) ; N^{\prime}(q, m)$, completing the proof for this case.

Since $\alpha, \mu, \varepsilon>0$, to prove (7) it suffices to take arbitrary atoms $a \leq \alpha$ and $b \leq \mu$, and find an atom $c \leq \varepsilon$ such that the cycle $(a, b, \breve{c})$ is not forbidden. Then, $a \leq c ; \breve{b} \leq \varepsilon ; \breve{\mu}$ and $b \leq \breve{a} ; c \leq \breve{\alpha} ; \varepsilon$, and since $a, b$ were arbitrary, (7) follows.

The proof is a tedious but not difficult case analysis. Take any atom $c \leq \varepsilon$. If $(a, b, \breve{c})$ is not forbidden, we are done. Suppose then that it is forbidden. This is because one of F1-F5 applies to $(a, b, \breve{c})$. We have already established I1 for $N^{\prime}$, so $\alpha, \mu, \varepsilon \leq 0$ ' and F1 does not apply. F2 does not apply either, because $\exists$ does not include green atoms under $\varepsilon$, so $c$ is not green. Therefore one of F3-F5 forbids $(a, b, \breve{c})$. We consider each of these rules in turn.

Suppose F3 forbids $(a, b, \breve{c})$. By $\exists$ 's strategy (6), $c$ is not yellow, so we must have $a=\mathrm{y}_{x}, b=\mathrm{y}_{y}$, and $c=\mathrm{r}_{z}$ for some distinct $x, y, z \in \Gamma$. But then, $\mathrm{W} \leq a ; b \leq \alpha ; \mu$. By I1 for $N^{\prime}, \beta, \breve{v} \leq 0$ ', so by Lemma $4.4,(\beta ; \breve{v}) \cdot \mathrm{W}>0$. So by $\exists$ 's strategy, $\varepsilon \cdot \bar{W}>0$. Let $\mathrm{w}_{i} \leq \varepsilon$. Then $\left(a, b, \breve{\mathrm{w}}_{i}\right)=\left(\mathrm{y}_{x}, \mathrm{y}_{y}, \breve{\mathrm{w}}_{i}\right)$ is not forbidden, as required.

The other possibilities are handled in a similar way. If $(a, b, \breve{c})$ is forbidden by $\mathrm{F} 4$, then again, as $\exists$ does not include $\mathrm{g}_{i}, \mathrm{y}_{x}$ atoms in $\varepsilon$, we must have $a=\mathrm{y}_{x}$ and $b=\mathrm{g}_{i}$, for some $i<4$ and $x \in \Gamma$, and $\breve{c} \in B_{x, i}$ (see (2) above for $B_{x, i}$ ), or, since green and yellow atoms are self-converse, $a=\mathrm{g}_{i}, b=\mathrm{y}_{x}$ and $c \in B_{x, i}$. So there are two cases:

CASE $a=\mathrm{g}_{i}$ AND $b=\mathrm{y}_{x}$ : let $j, k<4$ be the numbers of opposite parity to $i$. Then $\mathrm{w}_{j}+\mathrm{w}_{k} \leq \mathrm{g}_{i} ; \mathrm{y}_{x} \leq \alpha ; \mu$. Take arbitrary atoms $d \leq \beta, e \leq \breve{v}$ : see figure 5 .

If $\mathrm{w}_{j} \leq d ; e$, then by $\exists$ 's strategy $(6), \mathrm{w}_{j} \leq \varepsilon$. As $\left(a, b, \breve{\mathrm{w}}_{j}\right)=\left(\mathrm{g}_{i}, \mathrm{y}_{x}, \breve{\mathrm{w}}_{j}\right)$ is not forbidden, we are done. A similar argument can be made if $\mathrm{w}_{k} \leq d ; e$.

So suppose $\mathrm{w}_{j}, \mathrm{w}_{k} \not \leq d ; e$. Only rules F4 and F5 can block white atoms (recall that F1 is not involved). Suppose that $\mathrm{w}_{j}, \mathrm{w}_{k} \not \leq d$; $e$ because of F5, so $d=\mathrm{w}_{s}$ for some $s \neq j, k$, and $e=\mathrm{v}$. As in Lemma 4.5, pick $y \in E(x)$ (using that $\Gamma$ is connected and has at least two nodes because its chromatic number is at least 3 , so 


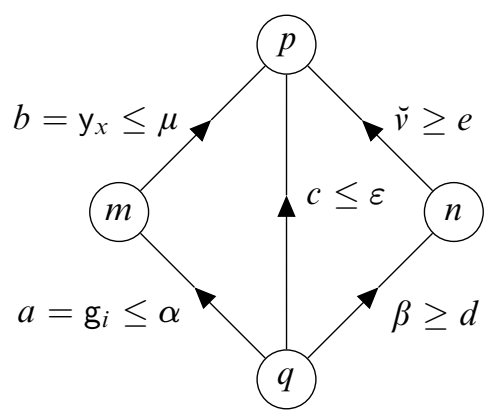

Figure 5. Atoms $a, b, c, d, e$.

that $E(x) \neq \emptyset)$. Then $\mathrm{r}_{y} \leq \mathrm{g}_{i} ; \mathrm{y}_{x} \leq \alpha ; \mu$ and also $\mathrm{r}_{y} \leq \mathrm{w}_{s} ; \mathrm{v} \leq \beta ; \breve{v}$. Therefore, $\exists$ 's strategy (6) ensured that $\mathrm{r}_{y} \leq \varepsilon$, and $\left(a, b, \breve{r}_{y}\right)=\left(\mathrm{g}_{i}, \mathrm{y}_{x}, \mathrm{r}_{y}\right)$ is not forbidden, as required.

Suppose instead that $\mathrm{w}_{j}, \mathrm{w}_{k} \not \leq d$; $e$ because of $\mathrm{F} 4$. This can only be because $d \in\left\{\mathrm{g}_{j}, \mathrm{~g}_{k}\right\}$ and $e=\mathrm{y}_{y}$ for some $y \in \Gamma$. Suppose without loss of generality that $d=\mathrm{g}_{j}$. But now, $k \neq i, j$, so we see (cf. F4) that $\breve{\mathrm{w}}_{k} \leq\left(\mathrm{g}_{i} ; \mathrm{y}_{x}\right) \cdot\left(\mathrm{g}_{j} ; \mathrm{y}_{y}\right)=(a ; b)$. $(d ; e) \leq(\alpha ; \mu) \cdot(\beta ; \breve{v})$. Because $a=\mathrm{g}_{i} \leq \alpha$ and $d=\mathrm{g}_{j} \leq \beta$, we have $\alpha \cdot \mathrm{G}>0$ and $\beta \cdot \mathrm{G}>0$. So $\exists$ would include $\breve{\mathrm{w}}_{k}$ in $\varepsilon$ (see (6)), and $\left(a, b, \mathrm{w}_{k}\right)=\left(\mathrm{g}_{i}, \mathrm{y}_{x}, \mathrm{w}_{k}\right)$ is not forbidden, as required.

CASE $a=\mathrm{y}_{x}$ AND $b=\mathrm{g}_{i}$ : then $\mathrm{w}_{j} \leq a ; b \leq \alpha ; \mu$ for all three $j \neq i$. If there is such a $j$ with $\mathrm{w}_{j} \leq \beta$; $\breve{v}$, we are done, since $\exists$ would include $\mathrm{w}_{j}$ in $\varepsilon$, and $\left(a, b, \breve{\mathrm{w}}_{j}\right)=\left(\mathrm{y}_{x}, \mathrm{~g}_{i}, \breve{\mathrm{w}}_{j}\right)$ is not forbidden, as required. So assume not. Only F5 can block three white atoms, so in fact $\mathrm{w}_{i} \leq \beta$ and $\mathrm{v} \leq v$. As before, if $y \in E(x)$ then $\mathrm{r}_{y} \leq \mathrm{y}_{x} ; \mathrm{g}_{i}=a ; b \leq \alpha ; \mu$ and also $\mathrm{r}_{y} \leq \mathrm{w}_{i} ; \mathrm{v} \leq \beta ; \breve{v}$. Therefore, $\exists$ would ensure that $r_{y} \leq \varepsilon$, and $\left(a, b, r_{y}\right)$ is not forbidden, as required.

Finally suppose that $(a, b, \breve{c})$ is forbidden by F5. $\exists$ never includes $\vee$ in $\varepsilon$, so there are only two possibilities:

- $a=\mathrm{w}_{i}, b=\mathrm{v}$, and $c=\mathrm{w}_{j}$ for distinct $i, j<4$, or

- $a=\mathrm{v}, b=\breve{\mathrm{w}}_{i}$, and $c=\breve{\mathrm{w}}_{j}$, for distinct $i, j<4$.

So $\mathrm{r}_{\Gamma} \leq a ; b \leq \alpha ; \mu$. By Lemma $4.5, \mathrm{r}_{\Gamma} \cdot(\beta ; \breve{v})>0$. By $\exists$ 's strategy, there is some $\mathrm{r}_{x} \leq \varepsilon$. Then $\left(a, b, \mathrm{r}_{x}\right)$ is not forbidden, as required.

So (7) is proved, and we have the result when $\{x, y, z\}=\{p, m, q\}$. The case when $\{x, y, z\}=\{p, n, q\}$ for some $q \in N \backslash\{m, n\}$ is handled analogously or follows by symmetry.

Finally suppose that $\{x, y, z\}=\left\{p, q, q^{\prime}\right\}$ for distinct $q, q^{\prime} \in N \backslash\{m, n\}$. We check that $N^{\prime}(x, y) \leq N^{\prime}(x, z) ; N^{\prime}(z, y)$, and this is the most interesting part of the proof. Write $\varepsilon=N^{\prime}(q, p), \varepsilon^{\prime}=N^{\prime}\left(q^{\prime}, p\right), \alpha=N(q, m), \alpha^{\prime}=N\left(q^{\prime}, m\right), \beta=N(q, n)$, and $\beta^{\prime}=N\left(q^{\prime}, n\right)$. We saw above that $\varepsilon, \varepsilon^{\prime}>0$, and $N^{\prime}\left(q, q^{\prime}\right)>0$ by inductive hypothesis I1. Take arbitrary atoms $a \leq N\left(q, q^{\prime}\right), b \leq \varepsilon$, and $c \leq \varepsilon^{\prime}$ (see figure 6). It is enough if we show that

$$
a \leq \varepsilon ; \breve{\varepsilon}^{\prime}, \quad b \leq a ; \varepsilon^{\prime}, \quad \text { and } \quad c \leq \breve{a} ; \varepsilon .
$$




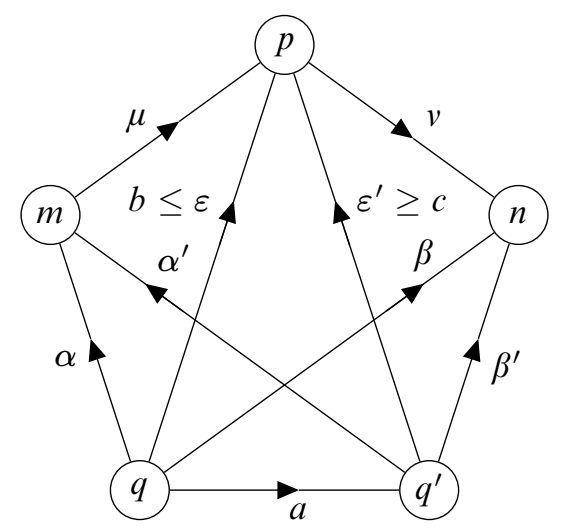

FIGURE 6. Points $m, n, p, q, q^{\prime} \in N^{\prime}$.

This is immediate if the cycle $(a, c, \breve{b})$ is not forbidden, so suppose that it is forbidden. We know by $\exists$ 's strategy that $b, c \leq \mathrm{r}_{\Gamma}+\mathrm{W}+\breve{\mathrm{W}}$, and $a \neq 1$ ' by inductive condition I1. Under these circumstances, only rule F5 can forbid the cycle $(a, c, \breve{b})$. So we must have

$$
a=\mathrm{v}, \quad b=\breve{\mathrm{w}}_{i}, \quad c=\breve{\mathrm{w}}_{j},
$$

for some distinct $i, j<4$. As $\exists$ has included atoms $\breve{\mathrm{w}}_{i}, \breve{\mathrm{w}}_{j}$ in $\varepsilon, \varepsilon^{\prime}$, we deduce from (6) that $\alpha, \alpha^{\prime}, \beta, \beta^{\prime}$ all contain green atoms.

Now there are essentially two cases. Suppose first that $\mu \not \leq \mathrm{y}_{\Gamma}$. Since $\alpha \cdot \mathrm{G}>0$, we see that $r_{\Gamma} \leq \alpha ; \mu$. By Lemma 4.5, $r_{\Gamma} \cdot(\beta ; \breve{v})>0$, and so there is some $r_{x} \leq \varepsilon$. Similarly, there is some $\mathrm{r}_{y} \leq \varepsilon^{\prime}$. But now we have $a=\mathrm{v} \leq \mathrm{r}_{x} ; \mathrm{r}_{y} \leq \varepsilon ; \breve{\varepsilon}^{\prime}$, as well as $b=\breve{\mathrm{w}}_{i} \leq \mathrm{v} ; \mathrm{r}_{y} \leq a ; \varepsilon^{\prime}$ and $c=\breve{\mathrm{w}}_{j} \leq \mathrm{v} ; \mathrm{r}_{x} \leq \breve{a} ; \varepsilon$, proving (8). The proof if $v \not \leq \mathrm{y} \Gamma$ is similar: again, red atoms creep into $\varepsilon, \varepsilon^{\prime}$.

So we pass to the other case, when $\mu, v \leq \mathrm{y}_{\Gamma}$. Say, $\mu=\mathrm{y}_{P}$ and $v=\mathrm{y}_{Q}$ for $P, Q \subseteq \Gamma$.

Claim 3. $E(P) \cap E(Q) \neq \emptyset$.

Proof OF Claim. First, if there is some $y \in P \cap Q$, then as before, $\emptyset \neq E(y) \subseteq$ $E(P) \cap E(Q)$ and we are done.

So suppose that $P \cap Q=\emptyset$. We know that $\mu, v>0$, so $P, Q \neq \emptyset$. Now the edge $(m, n)$ is plainly critical in $N$, and so by inductive assumption $\mathrm{I} 2, \mathrm{r}_{\Gamma} \leq N(m, n) \leq$ $\mu ; v=\mathrm{y}_{P} ; \mathrm{y}_{Q}$. By rule $\mathrm{F} 3$ and because $P \cap Q=\emptyset$, we obtain $P \cup Q=\Gamma$.

Our assumption that $\chi(\Gamma)>2$ has not been used yet. We use it now. Since $\chi(\Gamma)>2$, not both of $P, Q$ are independent. So one of them, say $P$, contains nodes $x, y$ such that $x y$ is an edge of $\Gamma$. Since $Q \neq \emptyset$, we can pick $t \in Q$. Since $\Gamma$ is connected, there is a path $x=x_{1}, x_{2}, \ldots, x_{n}=t$ from $x$ to $t$ in $\Gamma$, where $n>1$. Let $i<n$ be maximal such that $x_{1}, \ldots, x_{i} \in P$, and write $z=x_{i}$. Then $z x_{i+1}$ is an edge of $\Gamma$, and $x_{i+1} \in Q$, so $z \in E(Q)$. Also, letting $x_{0}=y$, clearly $x_{i-1} z$ is also an edge and $x_{i-1} \in P$, so $z \in E(P)$ as well. This proves the claim.

So take $z \in E(P) \cap E(Q)$. We know $\alpha \cdot \mathrm{G}, \beta \cdot \mathrm{G}>0$, so we may take green atoms $\mathrm{g}_{k} \leq \alpha$ and $\mathrm{g}_{l} \leq \beta$. By (inapplicability of) rule $\mathrm{F} 4$, we have $\mathrm{r}_{z} \leq\left(\mathrm{g}_{k} ; \mathrm{y}_{P}\right) ;\left(\mathrm{g}_{l} ; \mathrm{y}_{Q}\right) \leq$ 
$(\alpha ; \mu) \cdot(\beta ; \breve{v})$, and so by $\exists$ 's strategy, $r_{z} \leq \varepsilon$. Similarly, $r_{z} \leq \varepsilon^{\prime}$. But now, $a=\mathrm{v} \leq \mathrm{r}_{z} ; \mathrm{r}_{z} \leq \varepsilon ; \breve{\varepsilon}^{\prime}, b=\breve{\mathrm{w}}_{i} \leq \mathrm{v} ; \mathrm{r}_{z} \leq a ; \varepsilon^{\prime}$, and $c=\breve{\mathrm{w}}_{j} \leq \mathrm{v} ; \mathrm{r}_{z} \leq \breve{a} ; \varepsilon$, proving (8) again.

We have shown that $N^{\prime}$ is a network satisfying the inductive hypotheses. So we have provided a winning strategy for $\exists$ in the game $G(\mathscr{A})$. This completes the proof of Proposition 4.9.

§5. Conclusion. We proved that wRRA is not closed under completions and is not canonical. It follows [8] that wRRA is not generated as a variety by (the full complex algebras over) any elementary class of structures. We mention two open problems. First, is wRRA generated as a variety by any class of structures at all? In standard notation and terminology, this asks whether wRRA is a complete variety: whether there is a class $\mathrm{K}$ of atom structures such that $\mathrm{wRRA}=\boldsymbol{H} \boldsymbol{S P C m} \mathrm{K}$. Second, Goldblatt has asked whether wRRA is a complex variety: whether there is a class $\mathrm{K}$ of atom structures such that wRRA $=\boldsymbol{S C m K}$. Obviously, all canonical varieties are complex and all complex varieties are complete. Examples are known of complex non-canonical varieties [27] and of complete non-complex varieties [8, Theorem 3.7.1].

\section{REFERENCES}

[1] H. ANDRÉKA, Weakly representable but not representable relation algebras, Algebra Universalis, vol. 32 (1994), pp. 31-43.

[2] H. ANDRÉKA and D. A. BREDIKHIN, The equational theory of union-free algebras of relations, Algebra Universalis, vol. 33 (1995), pp. 516-532.

[3] H. Andréka, R. Goldblatt, and I. NÉmeti, Relativised quantification: Some canonical varieties of sequence-set algebras, this JournaL, vol. 63 (1998), pp. 163-184.

[4] P. Blackburn, M. de Rijke, and Y. Venema, Modal logic, Tracts in Theoretical Computer Science, Cambridge University Press, 2001.

[5] R. Diestel, Graph theory, Graduate Texts in Mathematics, vol. 173, Springer-Verlag, Berlin, 1997.

[6] M. Gehrke, J. Harding, and Y. Venema, MacNeille completions and canonical extensions, Transactions of the American Mathematical Society, vol. 358 (2006), pp. 573-590.

[7] R. Goldblatt, Metamathematics of modal logic, Reports on Mathematical Logic, vol. 6 (1976), pp. 41-77, reprinted in [9].

[8] - Varieties of complex algebras, Annals of Pure and Applied Logic, vol. 44 (1989), pp. 173242.

[9] - Mathematics of modality, Lecture notes, vol. 43, CSLI Publications, Stanford, CA, 1993.

[10] - Questions of canonicity, Trends in logic: 50 years of Studia Logica (Vincent F. Hendricks and Jacek Malinowski, editors), Kluwer Academic Publishers, 2003, pp. 93-128.

[11] - Mathematical modal logic: A view of its evolution, Handbook of the history of logic (D. M. Gabbay and J. Woods, editors), vol. 7, Elsevier, Amsterdam, 2006, pp. 1-98.

[12] M. Haiman, Arguesian lattices which are not linear, Bulletin of the American Mathematical Society, vol. 16 (1987), pp. 121-123.

[13] - Arguesian lattices which are not type I, Algebra Universalis, vol. 28 (1991), pp. 128-137.

[14] L. Henkin, J. D. Monk, and A. TARski, Cylindric algebras part II, North-Holland, 1985.

[15] R. Hirsch and I. Hodkinson, Relation algebras by games, Studies in Logic and the Foundations of Mathematics, vol. 147, North-Holland, Amsterdam, 2002.

[16] R. Hirsch, I. Hodkinson, and R. D. Maddux, Weak representations of relation algebras and relational bases, this JourNaL, vol. 76 (2011), pp. 870-882.

[17] I. Hodkinson, Atom structures of cylindric algebras and relation algebras, Annals of Pure and Applied Logic, vol. 89 (1997), pp. 117-148. 
[18] I. Hodkinson and Sz. MikUlás, Non-finite axiomatizability of reducts of algebras of relations, Algebra Universalis, vol. 43 (2000), pp. 127-156.

[19] B. Jónsson, Representation of modular lattices and of relation algebras, Transactions of the American Mathematical Society, vol. 92 (1959), pp. 449-464.

[20] B. JónsSON and A. TARSKI, Boolean algebras with operators I, American Journal of Mathematics, vol. 73 (1951), pp. 891-939.

[21] R. D. MADDUX, A sequent calculus for relation algebras, Annals of Pure and Applied Logic, vol. 25 (1983), pp. 73-101.

[22] - Relation algebras, Studies in Logic and the Foundations of Mathematics, vol. 150, North-Holland, Amsterdam, 2006.

[23] R. McKenZIE, The representation of relation algebras, Ph.D. thesis, University of Colorado at Boulder, 1966.

[24] J. D. Monk, Completions of boolean algebras with operators, Mathematische Nachrichten, vol. 46 (1970), pp. 47-55.

[25] B. PÉCSI, Weakly representable relation algebras form a variety, Algebra Universalis, vol. 60 (2009), pp. 369-380.

[26] A. M. Robert, A course in p-adic analysis, Graduate Texts, Springer, New York, 2000.

[27] F. Wolter, Properties of tense logics, Mathematical Logic Quarterly, vol. 42 (1996), pp. 481-500.

DEPARTMENT OF COMPUTING

IMPERIAL COLLEGE LONDON

LONDON, SW7 2AZ, UK

$U R L$ : www.doc.ic.ac.uk/ imh/

DEPARTMENT OF COMPUTER SCIENCE AND INFORMATION SYSTEMS

BIRKBECK, UNIVERSITY OF LONDON

LONDON. WCIE 7HX. UK

$U R L:$ www.dcs.bbk.ac.uk/ $\sim_{\text {szabolcs/ }}$ 